\title{
Quality of life among Brazilian women having undergone surgery for breast cancer: Validity and reliability of the Quality of life Questionnaire (EORTC QLQ-C30) and Breast Cancer Module (QLQBR-23)
}

\author{
Ana Silvia Diniz Makluf ${ }^{1}$, Alexandre de Almeida Barra ${ }^{2 *}$, Rosângela Corrêa Dias ${ }^{3}$ and Cristovão Pinheiro de Barros ${ }^{4}$ \\ ${ }^{1}$ Rehabilitation Sciences, Federal University of Minas Gerais, Brazil \\ ${ }^{2}$ Medicine in Federal University of Ouro Preto, Brazil \\ ${ }^{3}$ Physiotherapy Department, Physiotherapy and Occupational Therapy School, Federal University of Minas Gerais, Brazil \\ ${ }^{4}$ Oncology Service of the Instituto de Previdência dos Servidores do Estado de Minas Gerais, Brazil
}

\begin{abstract}
The aim of the present study was to verify the measurement properties of the generic Quality of Life Questionnaire (EORTC QLQ-C30) and specific Breast Cancer Module (QLQBR-23) for assessing the quality of life of Brazilian women having undergone breast cancer surgery. The measurement properties of the instruments were evaluated with regard to internal consistency, discriminant validity, criteria validity, and test-retest reliability. Adequate internal consistency indices $(\alpha=0.47$ to 085 ) were demonstrated. Stepwise multiple linear regression analysis was used to determine criteria validity, demonstrating that the domains for pain (PA), emotional functioning (EF) and social functioning (SF) explained $46 \%$ of the variance. The instruments presented satisfactory test-retest reliability in both the interobserver and intra-observer evaluations. The EORTC QLQ-C30 and BR-23 instruments proved adequate in assessing the quality of life of Brazilian women having undergone breast cancer surgery and also demonstrated the importance of assessing the quality of life as a health indicator with the aim of seeking more qualified and humanized care.
\end{abstract}

\section{Introduction}

Quality of life rests on the comprehension of fundamental, material and spiritual human needs. Its most relevant focus is on the concept of health promotion. Despite the consensus on the relation between health and quality of life, as well as its importance in health promotion, it is as yet uncommon to infer it in clinical practice [1].

Quality of life (QOL) has become a part of the evaluation criteria for cancer therapy besides the classical biomedical criteria. It is the most frequently used outcome measures in oncology research. The majority of clinical trials included QOL as a secondary endpoint in phase III investigations. Various instruments for measuring QOL of cancer patient have been developed [2].

Breast cancer is the second most frequent cancer type worldwide and the most frequent in women and the treatment, which is often mutilating, can lead to changes in a woman's self-image and functional loss as well as psychological, emotional and social alterations. Such alterations in women that have undergone treatment for carcinoma of the breast can be quantified on a quality of life scale [3].

No study has been located thus far that demonstrates the psychometric properties of an instrument designed to assess the quality of life of Brazilian women with breast cancer. Employing an instrument that is not adapted to our actuality has been the focus of various considerations and criticisms. The same is true regarding the creation of instruments that already exist in other languages, as such a procedure would impede comparisons with other studies carried out in different countries and among different populations.

The aim of the present study was to evaluate the measurement properties of the generic QLQ-C30 instrument and specific BR23 instrument for breast cancer, determining internal consistency, discriminant validity, criteria validity, and test-retest reliability, as well as assessing the quality of life of Brazilian women having undergone breast cancer surgery.

\section{Methods}

A descriptive, cross-sectional, correlational study was carried out involving sixty-seven women who were surgically treated for breast cancer. Patients having undergone adjuvant treatment prior to surgery, such as systemic treatment, and women with metastatic disease were excluded from the study.

Patients were selected randomly among those treated at the Instituto de Previdência dos Servidores do Estado de Minas Gerais (IPSEMG)

Correspondence to: Prof. Alexandre de Almeida Barra, Medicine in Federal University of Ouro Preto, Rua Padre Marinho 49, Sala 405, Belo Horizonte, Minas Gerais, Brazil, Cep: 30.140040, Tel: 5531 32413797/553191619368; E-mail: barraalexandre@hotmail.com

Key words: breast cancer, instrumentsm quality of life, measurement properties

Received: February 12, 2015; Accepted: March 05, 2015; Published: March 08, 2015 
from December 2002 to September 2004. This is a public hospital that have an important reference in medical and hospital care in Minas Gerais, Brazil. After surgical treatment for breast cancer, patients were sent from the surgeon to rehabilitation. The questionnaires were applied after approval from the Ethics and Medical Research Committee of IPSEMG, under the register of CEP- HGIP: 077/03. The EORTC QLQ-C30 and BR-23 instruments were administered to the entire sample an average of $18(12-33)$ days after surgical treatment.

Quality of life was measured after breast cancer surgery, using the Questionnaire of the European Organization for Research and Treatment of Cancer EORTC QLQC30 and the BR23 module (supplementary questionnaire specific for patients with breast cancer) [4-6].

The interview was conducted face-to-face by trained researcher. The EORTC QLQ-C30 is a validated questionnaire translated into Portuguese of Brazil. It is composed of five functional scales (covering the physical, role, emotional, cognitive, and social aspects), symptoms scales and items (fatigue, nausea and vomiting, pain, dyspnea, insomnia, appetite loss, constipation, diarrhea, financial difficulties), and a global health. The scores on QOL were calculated according to the rules established by the EORTC (range 0-100), through which low scores indicate better QOL for symptoms scales and higher results suggest better QOL scores for global health and functional scales. The BR23 has four functional scales (body image, future perspective, sexual functioning, and enjoyment) and symptom scales (systemic therapy side effects, breast and arms symptoms, upset by hair loss), with scores that are similar to C30 questionnaire.

To determine test-retest reliability, the EORTC QLQ-C30 and BR23 questionnaires were re-administered to 24 women an average of 5.6 days after the initial assessment. To test inter and intra-examiner reliability, an additional duly trained physiotherapist applied the questionnaire to 12 women, whereas one of the authors of the study re-administered the questionnaires to another 12 women.

Missing data can be classified as missing items (one or more questions left unanswered on a questionnaire) or missing forms (the patient fails to respond to the entire questionnaire) [7].

Patients often failed to answer questions referring to symptoms of systemic treatment (chemotherapy), such as diarrhea, vomiting, on the generic questionnaire and questions regarding sexuality and sexual pleasure on the specific QLQ BR-23 instrument, as the questionnaire was administered during the post-surgery period when such symptoms are not common and women also were not sexually active due to their condition.

According to the recommendations of the SCORING MANUAL 2001 [4], if at least half of the questions on the questionnaire are answered, the standard equation of the score calculation should be applied, ignoring the missing data. This procedure was adopted for questions in which missing data were recorded.

\section{Results}

The mean age of the women was 55.0 years SD 10.7. Regarding schooling, $32.8 \%$ of the women had an upper education. $56.7 \%$ were married and $35.8 \%$ of the women were retired. The majority of the patients $(76.1 \%)$ presented Stages I and II of the disease. Regarding type of surgery, $52.2 \%$ of the women had undergone conservative breast surgery and $82.1 \%$ had undergone axillary lymph node dissection (ALND) (Table 1).
Table 1. Descriptive variables of the study.

\begin{tabular}{|c|c|c|c|}
\hline Descriptive variables & & $\mathbf{n}$ & $\%$ \\
\hline \multirow{3}{*}{ Type of surgery } & Conservative & 35 & $52.20 \%$ \\
\hline & Mastect. with reconst & 23 & $34.30 \%$ \\
\hline & Mastect. without reconst & 9 & $13.40 \%$ \\
\hline \multirow[t]{2}{*}{ Axillary lymph node } & Axillary lymph node dissection & 55 & $82.10 \%$ \\
\hline & Sentinel lymph node & 12 & $17.90 \%$ \\
\hline \multirow[t]{4}{*}{ Important loss } & yes & 48 & $71.60 \%$ \\
\hline & no & 19 & $28.40 \%$ \\
\hline & illiterate & 1 & $1.50 \%$ \\
\hline & incomplete elementary & 17 & $25.40 \%$ \\
\hline \multirow[t]{5}{*}{ Schooling } & complete elementary & 4 & $6.00 \%$ \\
\hline & incomplete high school & 23 & $34.30 \%$ \\
\hline & upper education & 22 & $32.80 \%$ \\
\hline & Single & 13 & $19.40 \%$ \\
\hline & Married & 38 & $56.70 \%$ \\
\hline \multirow[t]{4}{*}{ Marital state } & Widowed & 8 & $11.90 \%$ \\
\hline & Separated & 3 & $4.50 \%$ \\
\hline & Divorced & 5 & $7.50 \%$ \\
\hline & I & 21 & $31.30 \%$ \\
\hline \multirow[t]{4}{*}{ Tumor stage } & II & 30 & $44.80 \%$ \\
\hline & III & 5 & $7.50 \%$ \\
\hline & No record & 9 & $16.40 \%$ \\
\hline & Capital & 27 & $40.30 \%$ \\
\hline \multirow[t]{3}{*}{ Residence } & Interior & 39 & $58.20 \%$ \\
\hline & No record & 1 & $1.50 \%$ \\
\hline & $350-600$ Reals & 10 & $14.90 \%$ \\
\hline \multirow[t]{2}{*}{ Family income } & $>600-1200$ Reals & 23 & $34.30 \%$ \\
\hline & $>1200$ Reals & 34 & $50.70 \%$ \\
\hline
\end{tabular}

Abbreviations: mastect.: mastectomy, reconst.: reconstruction

Cohabitation with husband, children and grandchildren was a descriptive variable considered in the present study and 95\% resided in some family arrangement. It was also determined if these women had experienced any important family, financial or emotional loss in the five years prior to the diagnosis; $71.6 \%$ of the women confirmed such a loss.

Internal consistency of the EORTC QLQ-C30 and BR-23 instruments was evaluated through Cronbach's alpha coefficient in all the domains of the scales that presented more than one question. The objective was to determine if the questions for the same domain measure the same factor and if they are correlated to one another.

Altmam [8] states that Cronbach's alpha coefficient has a direct interpretation and depends upon the type of study to which it is employed. To compare groups, $\alpha$ values between 0.7 and 0.8 are considered satisfactory, whereas values greater than $\alpha=0.95$ are desirable for clinical application. In a validity study of the EORTC QLQ-C30 instrument, Aaronson et al. [4] analysis performed using Cronbach's alpha coefficient demonstrated satisfactory characteristics of internal consistency for the EORTC QLQ-C30 and Br-23 instruments. In the generic EORTC QLQ-C30 instrument, the global health status domain (QL2) presented a value of $\alpha=0.82$. For the functional scale of the five domains tested, Cronbach's alpha coefficient ranged from $\alpha=0.47$ for role functioning to $\alpha=0.79$ for emotional functioning. For the symptom scale, the nausea/vomiting domain presented the lowest value $(\alpha=0.36)$, whereas the pain domain presented the highest value $(\alpha=0.73)$.

Regarding the specific QLQ BR-23 instrument, the body image domain for the functional scale presented a value of $\alpha=0.85$, whereas 
Makluf ASD (2015) Quality of life among Brazilian women having undergone surgery for breast cancer: Validity and reliability of the Quality of life Questionnaire (EORTC QLQ-C30) and Breast Cancer Module (QLQBR-23)

Table 2. Analysis of variance comparing groups of women with regard to type of surgery: conservative or mastectomy with and without immediate reconstruction.

\begin{tabular}{|c|c|c|c|c|c|}
\hline \multirow{2}{*}{ Domain } & \multicolumn{3}{|c|}{ Average (sd) } & \multirow{2}{*}{$\mathbf{F}$} & \multirow{2}{*}{ Value $\mathbf{p}$} \\
\hline & Conservative $(n=35)$ & Mastectomy without immediate reconstruction $(n=23)$ & Mastectomy with immediate reconstruction $(n=9)$ & & \\
\hline QL2 & $67.8(22.7)$ & $74.6(21.4)$ & $77.8(24.6)$ & 1.03 & 0.362 \\
\hline PF2 & $85.2(16.5)$ & $80.9(19.3)$ & $85.2(17.3)$ & 0.44 & 0.644 \\
\hline RF2 & $71.4(31.5)$ & $61.6(33.5)$ & $64.8(40.3)$ & 0.63 & 0.537 \\
\hline EF & $66.0(28.6)$ & $55.4(32.4)$ & $75.0(26.7)$ & 1.64 & 0.202 \\
\hline $\mathbf{C F}$ & $78.1(30.2)$ & $71.0(32.3)$ & $68.5(42.9)$ & 0.49 & 0.614 \\
\hline SF & $76.2(31.1)$ & $53.6(31.0)$ & $64.8(42.0)$ & 3.34 & 0.042 \\
\hline FA & $16.8(23.4)$ & $24.6(2.07)$ & $12.3(15.1)$ & 1.14 & 0.328 \\
\hline NV & $6.2(12.8)$ & $8.7(20.0)$ & $1.9(5.6)$ & 0.67 & 0.513 \\
\hline PA & $28.1(29.4)$ & $29.0(29.8)$ & $38.9(31.2)$ & 0.48 & 0.619 \\
\hline DY & $2.9(16.9)$ & $7.2(17.3)$ & $0.0(0.0)$ & 0.85 & 0.433 \\
\hline SL & $29.5(39.4)$ & $43.5(44.3)$ & $44.4(47.1)$ & 0.95 & 0.391 \\
\hline AP & $11.4(22.8)$ & $18.8(33.1)$ & $3.7(11.1)$ & 1.24 & 0.297 \\
\hline CO & $13.3(30.5)$ & $20.3(37.3)$ & $0.0(0.0)$ & 1.39 & 0.257 \\
\hline DI & $3.8(13.5)$ & $11.6(29.5)$ & $0.0(0.0)$ & 1.53 & 0.224 \\
\hline FI & $31.4(38.7)$ & $33.3(44.9)$ & $66.7(40.8)$ & 2.74 & 0.072 \\
\hline
\end{tabular}

Abbreviations: QL2: Global Health Status, PF2: Physical Functioning, RF2: Role Functioning, EF: Emotional functioning, CF: Cognitive Functioning, Sf: Social Functioning, Fa: Fatigue, NV: Nausea and Vomiting, PA: Pain, DY: Dyspneia, SL: Sleeplessness, AP: Loss of Appetite, Co: Constipation, DI: Diarrhea, FI: Financial Difficulties.

sexual functioning was $\alpha=0.78$. For the symptom scale, the breast symptom domain presented the lowest value ( $\alpha=0.54)$, arm symptoms was $\alpha=0.57$ and side effects of systemic therapy was $\alpha=0.67$.

The women were separated into three groups with regard to type of surgery: conservative breast surgery, mastectomy and mastectomy followed by immediate reconstruction. Analysis of variance (ANOVA) was conducted to determine if the women having undergone more aggressive surgery (mastectomy) presented a poorer quality of life (Table 2).

Through ANOVA, no significant difference was found in the scores with regard to the type of surgery, except in the social functioning domain (SF), for which the average of the conservative surgery group was higher than that of the mastectomy without immediate reconstruction group, constituting a statistically significant difference $(\mathrm{p}<0.05)$.

Multiple linear regression analysis was conducted. The dependent variable was the global health status (QL2). The aim was to determine to what extent the domains explained the quality of life construct. The stepwise model was employed for demonstrating a tendency of linear growth among observed values versus adjusted values, which is expected when the adjustment is adequate.

In the criteria validity, the pain (PA), emotional functioning (EF) and social functioning (SF) domains appear in the linear model that explains $46 \%$ of the variance $\left(\mathrm{R}^{2}\right)$ using multiple linear regression.

In order to assess test-retest reliability and both intra and interobserver reliability, the questionnaires were administered to the same women and average of 5.6 day following the initial application. Questionnaires were re-administered to 24 women (33\% of the sample), 12 in the intra-observer evaluation and 12 in the inter-observer evaluation. The Student- $t$ test for paired samples was employed to determine if there was a difference in the averages of the domains for both the intra and inter-observer evaluations.

When test-retest reliability was evaluated, there was no significant difference in the score averages of the generic and specific instruments in the inter-observer and intra-observer evaluation.

\section{Discussion}

The present study demonstrates that the EORTC QLQ-C30 and BR-23 instruments have adequate psychometric properties of validity and reliability in assessing the quality of life among Brazilian women having undergone breast cancer surgery.

All the domains tested present satisfactory internal consistence, as evaluated by Cronbach's alpha coefficient ( $\alpha \geq 0.7)$. In the validity study of the EORTC QLQ-C30 questionnaire, Cronbach's alpha reliability coefficient ranged from $\alpha=0.52$ to $\alpha=0.89$. However, in the present study, as well as the validity study of the EORTC QLQ-C30 instrument, the role functioning domain presented low internal consistency ( $\alpha=0.47$ and $\alpha=0.54$ respectively). This reflects the limitation of this item, which is composed of only two questions addressing housework and work-related activities. Aaronson et al. [4] suggest that questions that assess leisure activities and hobbies be included in future versions of the questionnaires.

In the multicentric validity study of the specific EORTC BR23 instrument [6], Cronbach's alpha coefficient varied between populations, with the Spanish population presenting the lowest values ( $\alpha=0.46$ to 0.94 ). However, the authors did not stipulate the values found for each domain. In the present study, values for Cronbach's alpha coefficient ranged from $\alpha=0.54$ to 0.85 .

Regarding discriminant validity, there was no significant difference of the score averages in the domains in relation to the type of surgery. Therefore, this variable did not significantly alter the average quality of life among post-operatory breast cancer patients in the present study. This may have been due to the fact that the impact of the breast cancer diagnosis a women feels immediately following surgery is much greater than the effects of surgery. If this evaluation were made six months after surgery, the results may have been different.

In the present study, the group of women having undergone conservative surgery presented a higher average in the social functioning (SF) domain when compared to the group of women having undergone mastectomy with or without immediate reconstruction. Patients submitted to mastectomy present a diminished body image, greater difficulties in sexual life, limitations at work and even changes in daily living habits $[9,10]$. 
One limitation is related to the fact that this study included a great number of women with an indication for axillary lymph node dissection, featuring a more aggressive surgical approach and, consequently, greater impairment in functional activities and labor in order to prevent lymphedema. The technique of the sentinel lymph node, a less aggressive surgical approach was applied only to twelve patients. These issues could have affected the HQOL some month after the treatment. Generally, women in early stages of cancer (or with no comorbid conditions) had better QLQ-C30 scores than those in advanced disease stages (or with comorbid conditions) [11].

In the criteria validity, the pain (PA), emotional functioning (EF) and social functioning (SF) appear in a linear regression model and explain $46 \%$ of the dependent variable, global health status (QL2). Thus, the present study demonstrates that the pain, emotional functioning and social functioning domains are determinants that influence quality of life. It was observed that these domains were relevant at the time the questionnaire was administered - in the immediate post-operatory breast cancer period, when pain is very much present and emotional fragility, the fear of a recurrence and fear of death alter the emotional state of these women. Furthermore, the stigma of the disease, prejudices and incapacity for work lead to a diminishing of their social functioning.

The majority of the patients (76.1\%) presented Stages I and II of the disease. The original intention was to assess quality of life after separating the women in terms of tumor stages, but as Stages I and II represent most of the diagnoses, it was not possible to conduct this analysis. Patients with larger tumours were probably treated more aggressively than those with smaller tumours and this can have a negative effect on their overall health [12].

Quality of life is a multidimensional construct that changes over time [13]. A satisfactory instrument for assessing quality of life must be sensitive to such changes, and it is possible that a change found in the average of the domains is due to the time factor.

Some evidencies show that in the professional experience of caring for women with breast cancer, it seems clear that stress, anxiety and traumatic events these women have experienced precede this diagnosis. With this in mind, the women were asked whether there was any important financial or family loss within a five-year period prior to the breast cancer diagnosis. A total of 48 women (71.6\%) reported having experienced a significant loss in their lives. Aguiar et al. [14] argue that social networking is crucial to deal with the psychosocial issues related to cancer and late effects of treatment, support in decision-making, and coping with the disease, in addition to providing emotional support, and reducing anxiety, stress, and sadness.

The choice of the QLQ-C30 and BR-23 instruments for measuring the quality of life among women with breast cancer was based on the fact that these instruments are widely referenced in the literature $[10,11,15]$ and are of easy application. The average application time for the Brazilian population is 14 minutes $( \pm 5.3)$ when the two instruments are combined (EORTC QLQ-C30 and BR-23). This application time is 9.2 minutes ( \pm 4.7) in English language-speaking countries [5]. Some authors related that it is necessary more time to apply the question naries. The average time to apply only the QLQ-BR 23 in the Moroccan patients was $15 \mathrm{~min}$ [15]. In the present study, the instrument was administered through an interview and not self-administered as in other countries. Interviews demand more time, which thereby explains this difference.
It was observed comprehension difficulty with Question 16 in the Portuguese version of the generic EORTC QLQ-C30 instrument "Have you been feeling obstipated?" and we suggest the modification of this question to "Have you been experiencing arrested intestine?", which is better adapted to the Brazilian Portuguese language widely used, leading to an ease of comprehension and application of the instrument to the Brazilian population.

Ferrel et al. [16,17] defined a multidimensional QOL model in cancer survivors that covered four dimensions: physical well-being, consisting of questions related to body and functional changes; psychological wellbeing, covering emotional distress, health concerns, future prospective, uncertainty, and vulnerability; social wellbeing, which refers to the social support, relationships, and financial condition; and spiritual well-being, which includes spirituality, meaning of illness experience, and ability to maintain hope. It may be noted that the dimensions presented by the author, although with a different approach to the object, are quite similar to the three components of QOL identified in this study.

The social networking is crucial to deal with the psychosocial issues related to cancer and late effects of treatment, support in decision-making, and coping with the disease, in addition to providing emotional support, and reducing anxiety, stress, and sadness [18,19].

The emotional factors such as stress, loss of a loved one, separation, or financial problems can have important effects upon physical health, aggravating certain morbid states and creating many others. This gives rise to the understanding that the mind and body, far from being distinct dimensions of human beings, are constantly interacting, with both mutually beneficial and detrimental effects. In the early $20^{\text {th }}$ century, the spectacular progress of investigation techniques and medical interventions diverted attention from the interference of psychological factors in the genesis of disease. Surgical management became focussed on cancer as a disease located in a specific part of the body, often neglecting its relevance as an aspect of the functioning of the human being as a whole.

However, from the 1960s, scientific studies began to appear showing that a healthy emotional life is a protective factor against the development of disease and plays an important role in positive developments after cancer treatment

On future perspectives, when considering variables related to psycho-emotional conditions, worse scores for these variables were associated with an increased risk of death after adjusting for staging, age at interview, followup of breast cancer treatment, and marital status.

Groenvold et al. [20] using the EORTC QLQ C30 questionnaire and Hospital Anxiety and Depression Scale (HADS), examined whether psychological distress in newly diagnosed breast cancer patients was associated with their survival, and found that emotional function (psychological distress) was a predictor of mortality.

Watson et al. [21] evaluated whether helplessness/hopelessness (measured by the Mental Adjustment to Cancer Scale-MAC) and depression (measured by HADS) would have an impact on survival and disease-free survival. They found a statistically significant association between these variables (adjusted HR=1.53, $95 \%$ CI 1.11-2.11).

Watson et al. [21] observed that cancer treatment itself had a minor influence on the survivors QOL, and that social conditions assumed greater importance, even reflecting upon one's mortality. Further investigations to examine the social aspects related to QOL and their 
association with the survival of breast cancer patients are needed in order to understand these relationships in more depth and to propose measures that could help to strengthen their social support. The public health polices should consider strategies that can improve the condition of women submitted to breast cancer treatment. It is important that health professionals can identify social questions that can be improved, aiming at better QOL and consequently survival.

Although the instruments present items that address various aspects of the disease and can be employed at different times during breast cancer treatment, such as the post-operatory period, or during complementary treatments, such as systemic and locoregional treatment, EORTC QLQ-C30 and BR-23 also appear to be very universal. Thus, during the interviews we observed that the instruments are unable to measure many feelings and anxieties such women experience after the diagnosis of breast cancer.

The results of this study make available reliable instruments for the Brazilian population that are able to measure the quality of life among women having undergone breast cancer.

In the final considerations, we point out the challenge of establishing a routine for quality of life assessment that addresses the day-to-day needs of health services. Assessing quality of life and considering the perceptions of patients enable the creation of health indicators for more qualified and humanized health care, as this type of approach addresses various dimensions of the disease.

\section{References}

1. Lee SJ, Earle CC, Weeks JC (2000) Outcomes research in oncology: history, conceptual framework, and trends in the literature. J Natl Cancer Inst 92: 195-204. [Crossref]

2. Goodyear M, Fraumeni M (1996) Incorporating quality of life assessment into clinical cancer trials. In: Spilker B (edn) Quality of Life and Pharmacoeconomics in Clinical Trials. Philadelphia, PA: Lipincott-Raven 1003-1013.

3. Velloso FSB, Barra AA, Dias RC (2011) Functional performance of upper limb and quality of life after sentinel lymph node biobsy for breast cancer treatment. Revista Brasileira de Fisioterapia 15: 146-153.

4. Aaronson NK, Ahnedzai S, Bergman B, Bullinger M, Cull A, et al. (1993) The European organization for research and treatment of cancer QLQ-C30: A quality-oflife instrument for use international clinical trials in oncology. J Natl Cancer Inst 85 : 365-376. [Crossref]

5. (2001) Scoring Manual, Brussels.

6. Sprangers MA, Groenvold M, Arraras JI, Franklin J, te Velde A, et al. (1996) The
European Organization for Research and Treatment of Cancer breast cancer-specific quality-of-life questionnaire module: first results from a three-country field study. $J$ Clin Oncol 14: 2756-2768. [Crossref]

7. Troxel AB, Fairclough DL, Curran D, Hahn EA (1998) Statistical analysis of quality of life with missing data in cancer clinical trials. Stat Med 17: 653-666. [Crossref]

8. Altman DG (1991) Practical Statistics for Medical Research London: 164-168.

9. Janni W, Rjosk D, Dimpfl TH, Haertl K, Strobl B, et al. (2001) Quality of life influenced by primary surgical treatment for stage I-III breast cancer-long-term follow-up of a matched-pair analysis. Ann Surg Oncol 8: 542-548. [Crossref]

10. Engel J, Kerr J, Schlesinger-Raab A, Sauer H, Hölzel D (2004) Quality of life following breast-conserving therapy or mastectomy: results of a 5-year prospective study. Breast $J$ 10: 223-231. [Crossref]

11. Luo N, Fones CS, Lim SE, Xie F, Thumboo J, et al. (2005) The European Organization for Research and Treatment of Cancer Quality of Life Questionnaire (EORTC QLQ-c30): validation of English version in Singapore. Qual Life Res 14: 1181-1186. [Crossref]

12. De Gournay E, Guyomard A, Coutant C, Boulet S, Arveux P, et al. (2013) Impact of sentinel node biopsy on long-term quality of life in breast cancer patients. Br J Cancer 109: 2783-2791. [Crossref]

13. Spagnola S, Zabora J, BrintzenhofeSzoc K, Hooker C, Cohen G, et al. (2003) The Satisfaction with Life Domains Scale for Breast Cancer (SLDS-BC). Breast J 9: 463471. [Crossref]

14. De Aguiar SS, Bergmann A, Mattos IE (2014) Quality of life as a predictor of overall survival after breast cancer treatment. Qual Life Res 23: 627-637. [Crossref]

15. El Fakir S, Abda N, Bendahhou k (2014) The european organization for research and treatment of cancer quality of life questionnaire: psychometric properties in a Moroccan sample of breast cancer patients. BMC Research Notes 53: 1-7.

16. Ferrell BR, Hassey Dow K (1997) Quality of life among long-term cancer survivors. Oncology (Williston Park) 11: 565-568, 571. [Crossref]

17. Ferrell BR, Grant MM, Funk B, Otis-Green S, Garcia N (1997) Quality of life in breas cancer survivors as identified by focus groups. Psychooncology 6: 13-23. [Crossref]

18. Reynolds P, Boyd PT, Blacklow RS, Jackson JS, Greenberg RS, et al. (1994) The relationship between social ties and survival among black and white breast cancer patients. Cancer Epidemiol Biomarkers Prev 3: 253-259. [Crossref]

19. Chou AF, Stewart SL, Wild RC, Bloom JR (2012) Social support and survival in young women with breast carcinoma. Psychooncology 21: 125-133. [Crossref]

20. Groenvold M, Petersen MA, Idler E, Bjorner JB, Fayers PM, et al. (2007) Psychological distress and fatigue predicted recurrence and survival in primary breast cancer patients. Breast Cancer Res Treat 105: 209-219. [Crossref]

21. Watson M, Homewood J, Haviland J, Bliss JM (2005) Influence of psychologica response on breast cancer survival: 10 -year follow-up of a population-based cohort. Eur J Cancer 41: 1710-1714. [Crossref]

Copyright: (C) 2015 Salmo EN. This is an open-access article distributed under the terms of the Creative Commons Attribution License, which permits unrestricted use, distribution, and reproduction in any medium, provided the original author and source are credited. 